\title{
Planung operativer Eingriffe am Beckenring und Azetabulum
}

\author{
Axel Gänsslen, Hans-Jörg Oestern
}

\section{Zusammenfassung}

Die Planung von Osteosynthesen am Beckenring sowie bei Azetabulumfrakturen stellt höchste Anforderungen an Kenntnisse der knöchernen Anatomie, der Expositionsmöglichkeiten über die anzuwendenden Zugänge sowie die damit verbundenen Möglichkeiten, die dislokationsabhängige Reposition durchzuführen. Bei Beckenringverletzungen umfasst die Planung als wesentliche Schritte die Analyse der Verletzungsregionen, die Instabilitätsanalyse, die Fehlstellungsanalyse, die Klassifikation der Verletzung mit instabilitätsorientierter Auswahl des Osteosynthesekonzepts sowie die Auswahl der operativen Zugänge. Bei Osteosynthesen von Azetabulumfrakturen beinhaltet die Planung ausgedehnte Kenntnisse der Anatomie des knöchernen Hemipelvis, die Klassifikation der Fraktur mit Frakturmorphologie und die Auswahl des operativen Zugangs.

\section{Planning Surgical Interventions on the Pelvic Ring and Acetabulum}

The planning for internal fixation of pelvic ring injuries and acetabular fractures requires an extensive knowledge of the bony anatomy, the amount of possible exposure of the bone with the selected approaches and the resultant potential of displacement-dependent reduction. In pelvic ring injuries, planning includes as essential steps the assessment of the different fracture regions, an analysis of pelvic ring instability and the vectors of displacement, the classification of the injury leading to an instability-dependent osteosynthesis concept and the selection of surgical approaches. The planning for acetabular fracture fixation includes extensive knowledge of the anatomy of the bony hemipelvis, the classification of the fracture with detailed analysis of the fracture morphology and the choice of surgical approach.

\section{Einleitung}

Die Planung operativer Eingriffe am Beckenring und Azetabulum erfordert eine exakte Kenntnis der ossären und radiologischen Anatomie der Beckenregion und beinhaltet zusätzlich die Kenntnis und Dimension der einzusetzenden Implantate und Repositionshilfen.

Über die osteoligamentäre Anatomie des Beckenrings sowie die Besonderheiten der knöchernen Anatomie des Azetabulums wird auf die entsprechenden Übersichten in den Fachbüchern verwiesen.

OP-JOURNAL 2011; 27: 54-61

(c) Georg Thieme Verlag KG Stuttgart · New York DOI http://dx.doi.org/10.1055/s-0030-1271151
Im Folgenden sollen wichtige Schritte in der Beurteilung der Frakturen des Beckenrings und des Azetabulums, die zur Entscheidungsfindung beitragen, dargestellt werden.

Es sprengt das Ausmaß dieser Übersicht, detailliert auf mögliche operative Zugänge am Beckenring einzugehen. Für das Azetabulum werden die grundlegenden Kenntnisse der operativen Standardzugänge vorausgesetzt.

\section{Planung der operativen Versorgung von Beckenringfrakturen}

Die Planung der operativen Versorgung von Beckenringfrakturen orientiert sich an folgenden Aspekten:

- Analyse der Verletzungsregionen

- Instabilitätsanalyse
- Fehlstellungsanalyse

- Frakturklassifikation nach AO/OTA und Auswahl des Osteosynthesekonzepts

- Auswahl der operativen Zugänge

\section{Analyse der Verletzungsregion}

Im 1. Schritt der Planung der operativen Versorgung von Beckenringfrakturen ist die detaillierte Analyse der Verletzungsregionen anhand der Beckenübersichtsaufnahme und der Computertomografie (CT) notwendig.

Analyse der Verletzungsregionen nach Letournel.

Hierzu wird entsprechend der Vorgaben von Letournel jede mögliche Frakturregion analysiert. Im 1 . Schritt muss allerdings analysiert werden, ob das Becken streng anterior-posterior getroffen wurde. Dazu wird eine Linie in Verlängerung der Dornfortsätze der LWS in die Mitte des Sakrums gezeichnet und analysiert, ob diese Linie mittig durch die Symphyse verläuft. Nur dann können Fehlstellungen im Seitenvergleich der beiden Hemipelves sicher analysiert werden.

Als Standardröntgendiagnostik ist die Beckenübersichtsaufnahme (Abb. 1) im anterior-posterioren Strahlengang durchzuführen. Grobe Fehlstellungen bzw. Frakturen oder Gelenksprengungen lassen sich für fast alle Beckenringregionen nachweisen, allerdings lassen sich keine detaillierten Angaben über eine Verschiebung der Fragmente in anteriorposteriorer oder kraniokaudaler Richtung machen. Ergänzend können Schrägaufnahmen (Inlet-/Outlet-Aufnahmen) des Beckens angefertigt werden.

Die Inlet-Aufnahme erlaubt dabei eine genaue Beurteilung der Beckeneingangsebene. Ein ossärer Versatz nach dorsal oder ventral ist auf dieser Aufnahme gut zu beurteilen. Rechtwinklig dazu liegt die Projektion der Outlet-Aufnahme. 
Sie lässt besonders Vertikalverschiebungen gut zur Darstellung kommen.

Beide Aufnahmen sind jedoch durch die zunehmende präoperative Diagnostik mittels Computertomografie (CT) in den Hintergrund getreten. Da die intraoperative Beurteilung aber häufig auf diese beiden Aufnahmen zurückgreift, ist die Fehlstellungsanalyse mittels Inlet-/Outlet-Aufnahmen notwendig.

Die CT stellt heutzutage das Standardverfahren zur Detaildiagnostik dar. Die Möglichkeit, sekundär rekonstruierte Schnittebenen und 3-dimensionale Darstellungen $\mathrm{zu}$ generieren, erhöht die räumliche Vorstellbarkeit des Befunds erheblich. Die 3-dimensionale computertomografische Darstellung erlaubt eine ausgezeichnete anatomische Orientierung mit guter räumlicher Zuordnung der einzelnen Frakturen. Die Richtung der Dislokation von Fragmenten ist direkt erkennbar. Nachteile entstehen bei dieser Sichtweise bei der Feinbeurteilung.

Danach erfolgt die Analyse der Verletzungsregionen.

Symphysenverletzungen werden als reine distrahierende Gelenksprengungen oder selten als überlappende Symphysensprengungen gefunden. Als diagnostische Hilfslinien dienen die Linea iliopectinea, der Arcus pubis und die Symphysenbegrenzungslinien.

Die Linie des oberen Schambeinasts wird im Seitenvergleich analysiert. Es muss auf einen „Höhenversatz“ bzw. eine Asymmetrie geachtet werden. Der Arcus pubis kann durch Symmetrieanalyse Hinweise auf eine Verletzung geben. Der laterale Symphysenrand und damit der mediale Rand der Schambeine werden auf Parallelität überprüft.

Bei den sog. „open-book“-Verletzungen des Beckens mit begleitender Symphysensprengung und partieller bzw. kompletter Sprengung eines Sakroiliakalgelenks (ISG) kann radiologisch häufig eine typische radiologische Trias dargestellt werden (Abb. 1).

Radiologische Trias der „open book“-Verletzung.

Die verletzte Beckenhälfte zeigt im Symphysenbereich ein „Tiefertreten“ des Schambeinsegments gegenüber der Gegenseite, eine Außenrotationsfehlstellung des betroffenen Hemipelvis im Sei-

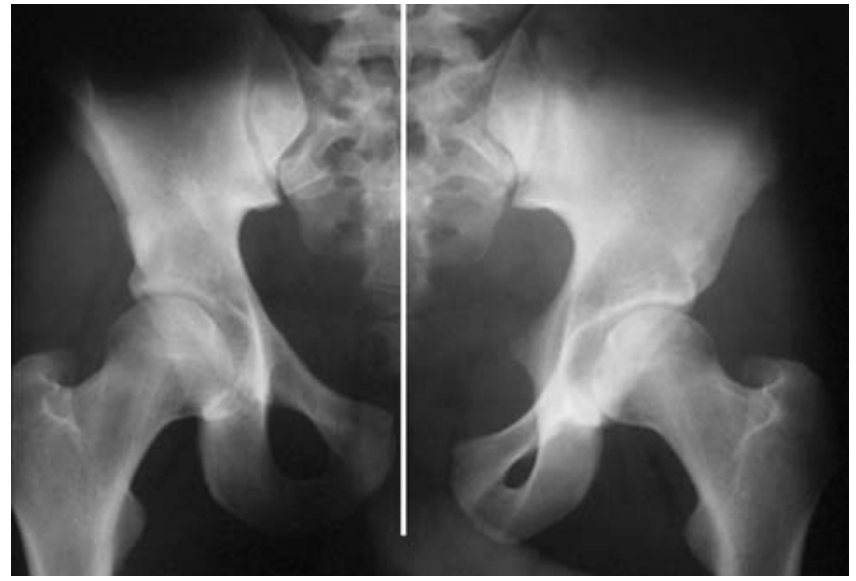

Abb. 1 Beckenübersichtsaufnahme mit eingezeichneter Mittellinie. Linksseitig besteht die klassische Fehlstellung der "open book"-Verletzung mit „Tiefertreten" des Schambeinsegments, Außenrotationsfehlstellung mit verbreiterter Beckenschaufel sowie Asymmetrie des Foramen obturatum.

tenvergleich, sichtbar durch eine verbreiterte Beckenschaufel sowie durch eine Asymmetrie des Foramen obturatum aufgrund der veränderten Rotation der Beckenhälfte.

Beurteilung der Linea iliopectinea für Schambeinastfrakturen.

Schambeinastfrakturen weisen sowohl konventionell radiologisch als auch im CT keine Probleme in der Diagnostik auf. Die wesentliche Kennlinie in der Beurteilung ist die aus der Diagnostik von Azetabulumfrakturen bekannte Linea iliopectinea, entsprechend der Linie des oberen Schambeinasts. Die Abgrenzung von "hoch“ auslaufenden Frakturen des oberen Schambeinasts zu Frakturen des Azetabulums gelingt mit letzter Sicherheit nur in der Computertomografie.

Analyse der Crista iliaca bei Iliumfrakturen.

Komplette Iliumfrakturen können diagnostische Schwierigkeiten bieten, da sie häufig nur gering disloziert sind, oder aufgrund der in der anterior-posterioren Ebene auf der Beckenübersichtsaufnahme vorliegenden Überlagerung verschiedener Strukturen des Iliums die eigentliche Frakturebene häufig nur schlecht und damit mit geringer Fehlstellung darstellbar ist. Analysiert werden die Linie der Crista iliaca, die proximale Linea iliopectinea und das dorsale Os ilium, welches sich hinter das Sakrum projiziert. Das CT zeigt hier sicher den Frakturverlauf und die entsprechende Fehlstellung sowie das Vorhandensein einer transiliakalen oder transsakralen Luxationsfraktur.

Analyse der ventralen und dorsalen Kennlinie des ISG.
Verletzungen des Iliosakralgelenks (ISG) können konventionell radiologisch anhand der ventralen Kennlinie (Spalt am Übergang der Linea iliopectinea zum Os sacrum) und der dorsalen Kennlinie (medial zur ventralen Kennlinie) analysiert werden. Die Linien werden primär auf Parallelität untersucht.

Bei nur rotationsinstabilen ISG-Sprengungen vom Typ B (s.u.) mit erhaltenen Ligg. sacroiliacalia dorsalia kommt es radiologisch zu einer Aufweitung der ventralen ISG-Anteile, die dorsalen ISGLinien verbleiben intakt (Abb. 1). Die betroffene Beckenhälfte rotiert nach außen, was sich in einer Verbreiterung des Iliums im Seitenvergleich zeigt.

Bei ISG-Sprengungen vom Typ C mit kompletter Zerreißung der ligamentären Strukturen weist die ventrale Kennlinie eine weite Diastase auf, in der Regel $>10 \mathrm{~mm}$, die dorsale Kennlinie ist nicht mehr nachweisbar. Neben der Diastase nach lateral kann auch ein Höhenversatz bzw. ein Versatz in der anterior-posterioren Ebene im Sinne einer „vertical shear"-Verletzung vorliegen. Rotationen um alle 3 möglichen Achsen kommen vor. Da das Becken „schräg“ im Raum geröntgt wurde, ist die wahre Fehlstellung nur anhand der CT analysierbar.

Analyse der Linea arcuarta bei Sakrumfrakturen

Die wesentliche Kennlinie in der Beurteilung von Sakrumfrakturen ist konventionell-radiologisch die Linea arcuarta (Abb. 2). Selbst kleine Verwerfungen dieser Linie beweist nahezu sicher eine Fraktur. Als weitere Hilfslinien dienen die Analyse der Sakrumschulter (Oberrand des Sakrums) sowie der Abstand von der Mittellinie des Sakrums zur Spitze der Sakrumschulter im Seitenvergleich. 


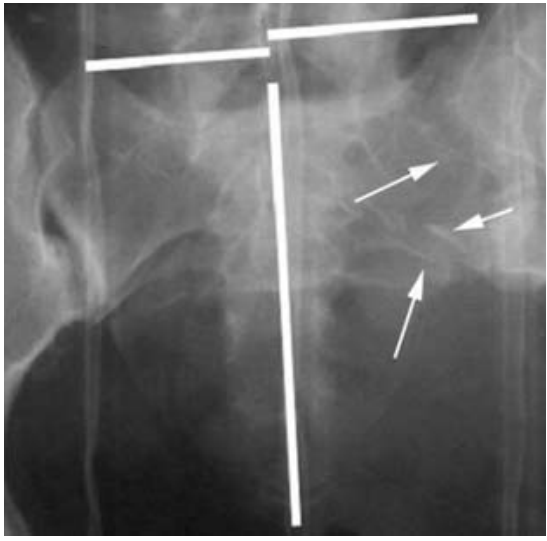

Abb. 2 Fraktur des linksseitigen Sakrums. Der Abstand zwischen Mittellinie und höchstem Punkt der Sakrumschulter ist links verbreitert. Die Unregelmäßigkeit an der Linea arcuarta links weist auf die Fraktur hin.

Die Analyse des Frakturverlaufs im Sakrum bleibt jedoch Domäne der CT-Diagnostik.

\section{Instabilitätsanalyse}

Im 2. Schritt muss nun entschieden werden, wie instabil die Beckenringverletzung ist.

Instabilitätsanalyse anhand des Ausmaßes der Beteiligung des hinteren Beckenrings

Durch die zunehmende Anwendung der CT zur primären Diagnostik von Beckenringverletzungen ist es in den letzten Jahren zu einer Verschiebung der Inzidenz von stabilen Typ-A-Verletzungen zu den rotationsinstabilen Typ-B-Verletzungen gekommen.

Bei Verletzungen vom Typ A ist der hintere Beckenring intakt. Frakturen liegen ausschließlich im Bereich des vorderen Beckenrings (seltener) oder betreffen den Beckenrand (Beckenschaufelfrakturen, Sakrumfrakturen unterhalb der Beckenringebene [S3-S5]).

Bei den rotationsinstabilen Beckenringverletzungen ist der dorsale Beckenring nur partiell unterbrochen. Eine Instabilität besteht um eine vertikale oder auch quere Achse. Hier kommt der detaillierten CT-Analyse entscheidende Bedeutung zu zur Differenzierung einer kompletten oder inkompletten Verletzung dorsal.

Bei Typ-C-Verletzungen ist der hintere Beckenring komplett unterbrochen, die Kontinuität zwischen allen osteoliga-
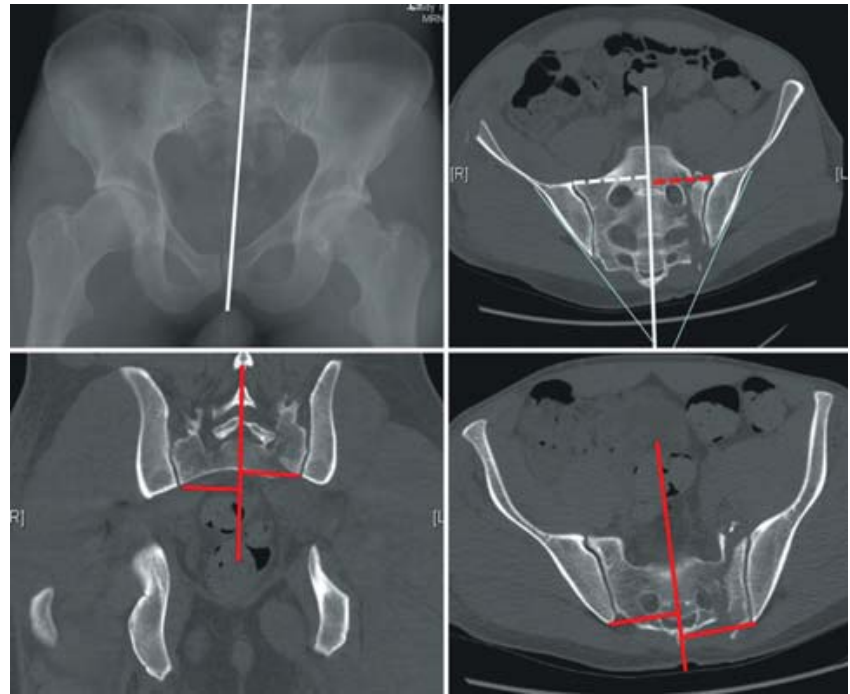

Abb. 3 Vermessung der Fehlstellung bei Sakrumfraktur. Mit verschiedenen Hilfslinien können ein Dorsalersatz, eine Kompression und eine Rotationsfehlstellung im CT objektiviert werden (s. Text).

mentären Elementen des hinteren Beckenrings ist verloren und damit eine 3-dimensionale translatorische und rotatorische Instabilität vorhanden. Die Beckenringverletzung ist instabil.

\section{Fehlstellungsanalyse}

Für die weitere Planung ist es nun wichtig, das Ausmaß der Fehlstellung (Dislokation), insbesondere am hinteren Beckenring, zu analysieren und damit die möglichen Repositionsmanöver $\mathrm{zu}$ planen. Hier hat sich die CT-gesteuerte Analyse der Fehlstellung bewährt.

Fehlstellungsanalyse mittels Hilfslinien in 3 Ebenen.

Zur Beurteilung der Fehlstellungen im hinteren Beckenring wird auf der Beckenübersichtsaufnahme, ggf. auch auf den Inlet- und Outlet-Aufnahmen, die Mittellinie am Sakrum eingezeichnet. Im CT sind entsprechende multiplanare Reformationen mit strenger a.-p. Ansicht auf das Sakrum bzw. senkrechter Reformation zur Beckeneingangsebene zu generieren (Abb. $\mathbf{3}$ ).

Zur Analyse des Kranialversatzes wird auf der Outlet-Aufnahme oder einer entsprechenden CT-Schicht im rechten Winkel dazu eine Tangente an die kranialsten Punkte des rechten und linken Beckenkamms von dieser Linie aus angelegt und der Abstand beider Schnittpunkte als Fehlstellung angenommen.

Ein Dorsalversatz wird auf der Inlet-Aufnahme oder einer entsprechenden CTSchicht im rechten Winkel zur Mittellinie als Tangente an die dorsalsten
Punkte des rechten und linken Beckenkamms von dieser Linie aus angelegt.

Weiter kann die Rotationsfehlstellung des verletzten Hemipelvis analysiert werden. Auch dazu wird die Mittellinie des Sakrums eingezeichnet. Hierbei ist entscheidend, dass die Reformationsebene derart gewählt wird, dass beidseits das dorsale Ilium auf gleicher Höhe angeschnitten ist. Es kann nun eine Tangente an die äußere Iliumkortikalis angelegt werden und im Seitenvergleich der entsprechende Winkel gemessen werden. Damit kann eine Innenrotations- oder Außenrotationsfehlstellung analysiert werden.

Eine mediolaterale Kompression oder Distraktion wird vergleichbar gemessen. Dazu wird von der Mittellinie jeweils eine Senkrechte zum ventralsten Punkt des ISG gezeichnet. Die beiden Abstände werden entsprechend gemessen und verglichen.

Entsprechend kann am vorderen Beckenring vorgegangen werden.

Die 3-D-Darstellung des Beckens erleichtert in einigen Fällen die genaue Analyse (Abb. 4).

\section{Klassifikation nach AO/OTA und Auswahl des Operationskonzepts}

Zusammenfassend erfolgt aufgrund der deskriptiven Frakturanalyse, der Fehlstellungsanalyse und der Instabilitätsanalyse die definitive Einschätzung der Fraktur und die entsprechende Zuordnung in eine der 3 Klassifikationstypen $(A, B, C)$. 


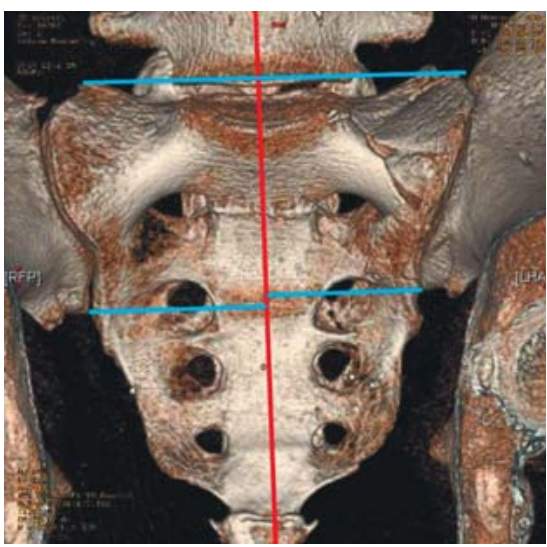

Abb. 4 Vermessung von Kompression und Höhenversatz im 3-D-CT (s. Text).

\section{Beckenringklassifikation nach AO/OTA.}

Die Einteilung in die Klassifikationsgruppen orientiert sich an der Fehlstellung und Instabilität.

Anhand der getroffenen Einteilung in die Frakturtypen A, B oder C erfolgt die prinzipielle Wahl des Osteosynthesekonzepts. Die Therapie der Beckenringverletzung orientiert sich v.a. am Instabilitätsgrad sowie am Ausmaß der Dislokation von Fragmenten.

Für die Typ-A-Verletzungen wird regelhaft ein nicht operatives Vorgehen empfohlen. Nur bei ausgedehnter Fragmentdislokation und Gefahr von Organverletzungen kommen operative Stabilisierungen zur Anwendung.

Für die rotationsinstabilen Typ-B-Verletzungen hat sich aufgrund des dorsal verbliebenen Scharniereffekts die ausschließlich ventrale Beckenringstabilisierung bewährt.

Für die dorsal komplett instabilen Typ-CVerletzungen werden mittel- und langfristig die optimalsten Ergebnisse durch kombinierte ventrale und dorsale Rekonstruktion des Beckenrings erreicht.

\section{Auswahl der operativen Zugänge}

Je nach Verletzungslokalisation wird der operative Zugang gewählt. Hier haben sich bestimmte Stabilisierungskonzepte mit entsprechenden Zugängen bewährt. Die Symphysenverplattung erfolgt über einen Pfannenstil-Zugang, ggf. erweitert als sog. Stoppa-Zugang.

Stabilisierungen dislozierter Iliumfrakturen oder ISG-Sprengungen werden
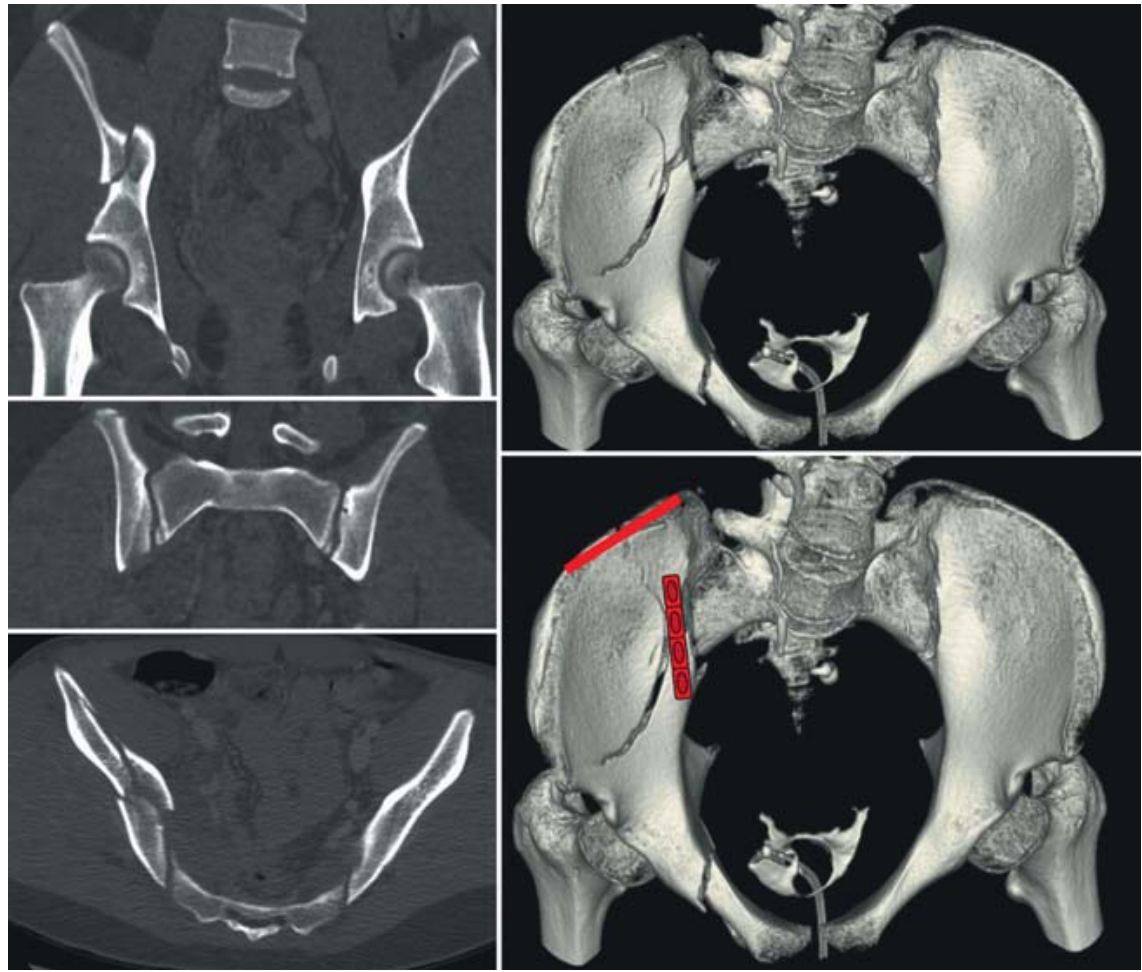

Abb. 5 Planung einer dorsalen Beckenringosteosynthese bei transiliakaler Luxationsfraktur (Details: s. Text).

meist über einen anterolateralen Zugang und mittels Plattenosteosynthese oder Schraubenosteosynthese versorgt.

Dislozierte Sakrumfrakturen erfordern häufig eine Reposition unter Sicht über einen dorsal medianen Zugang. Hier kommen verschiedene Stabilisierungsverfahren zur Anwendung.

Bezüglich der notwendigen Repositionsmanöver wird auf die entsprechende Fachliteratur verwiesen.

Beispiel der Planung einer Beckenringosteosynthese (Abb. 5)

16-jähriges Mädchen nach Pferdesturz mit Becken-C-Verletzung. Rechtsseitige transiliakale Luxationsfraktur sowie vordere Beckenringfraktur. Im Rahmen der Notfalltherapie erfolgte die Stabilisierung mittels supraazetabulärem Fixateur externe.

Die Analyse des CT inkl. 3-D-CT ergab folgenden Plan: aufgrund der nur minimal ausgeprägten Fehlstellung sowohl für Rotation, Kranial- und Dorsalversatz offene Reposition und interne Fixation des Os ilium über einen anterolateralen Zugang mit Verschraubung der Crista iliaca und Plattenosteosynthese im Bereich des Linea-terminales-nahen Iliums.

\section{Planung der operativen Versorgung von Azetabulumfrakturen}

Die Planung der operativen Versorgung von Azetabulumfrakturen orientiert sich an folgenden Aspekten:

- Anatomie des knöchernen Hemipelvis

- Frakturklassifikation nach Letournel

- Wahl des operativen Zugangs

- Frakturmorphologie

\section{Anatomie des knöchernen Hemipelvis}

Das ausgereifte Os coxae entspricht morphologisch einer in sich um etwa $90^{\circ}$ gewundenen Ziffer 8 (Abb.6). Innerhalb und unterhalb des Kreuzungspunkts der „Ziffer 8“ ist das Azetabulum eingesetzt. Wesentliche, Stabilität gebende knöcherne Strukturen finden sich jeweils am Rand dieser „Kreise“, im Zentrum fehlt der Knochen im Bereich des Foramen obturatum vollständig oder ist im Bereich der Fossa iliaca nur millimeterdünn bzw. kann bei muskelschwachen Individuen auch vollkommen fehlen. Die Kenntnis dieser 3-dimensionalen Ausrichtung und knöchernen Strukturverteilung ist wesentlich für die „traumatologische Anatomie“. Diese Vorstellungsweise erleichtert die Orientierung und lässt die zur Implantatverankerung nötigen „sicheren“ Schraubenrichtungen besser bestimmen. 


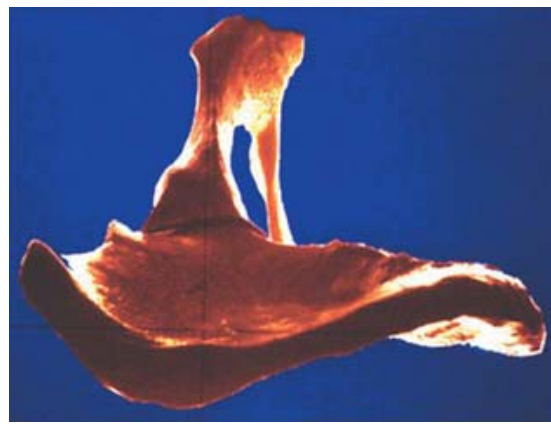

Abb. 6 Räumliche Orientierung von Obturatorregion und lliumregion.

Den besten Halt erhalten Implantate am Rand der beschriebenen Kreise.

\section{Frakturklassifikation nach Letournel}

Grundlage zur Planung der operativen Versorgung von Azetabulumfrakturen ist die Frakturklassifikation nach Letournel.

Anatomische Grundlage ist dabei die Pfeilerstruktur des Azetabulums (Abb. 7). Das Azetabulum besteht dabei funktionell aus einem vorderen und hinteren Pfeiler in Form eines auf dem Kopf stehenden Buchstabens „Y“, bei dem im Punkt des Zusammentreffens der beiden Schenkel die Hüftpfanne eingefügt ist.

Die konventionelle radiologische Diagnostik mittels Beckenübersichtsaufnahme und den Schrägaufnahmen nach Judet (Ala- und Obturatoraufnahme) ist der Standard der radiologischen Diagnostik zur Frakturklassifikation.

Durch Analyse verschiedener radiologischer Kennlinien auf der Beckenübersichtsaufnahme sowie den Schrägaufnahmen nach Judet werden 5 einfache, elementare Frakturtypen und 5 aus den elementaren Frakturtypen zusammengesetzte Frakturtypen unterschieden.

Bei den elementaren Frakturen werden Frakturen der hinteren Wand, Frakturen des hinteren Pfeilers, Frakturen der vorderen Wand, Frakturen des vorderen Pfeilers und Querfrakturen, die beide Pfeiler betreffen, unterschieden (Abb. 8).

Die zusammengesetzten Frakturtypen werden in Frakturen des hinteren Pfeilers mit Fraktur der hinteren Wand, Querfrakturen mit Frakturen der hinteren Wand, T-Frakturen, Frakturen der vorderen Wand und/oder des vorderen Pfeilers mit hinterer Hemiquerfraktur und ZweiPfeiler-Frakturen unterteilt (Abb. 8).

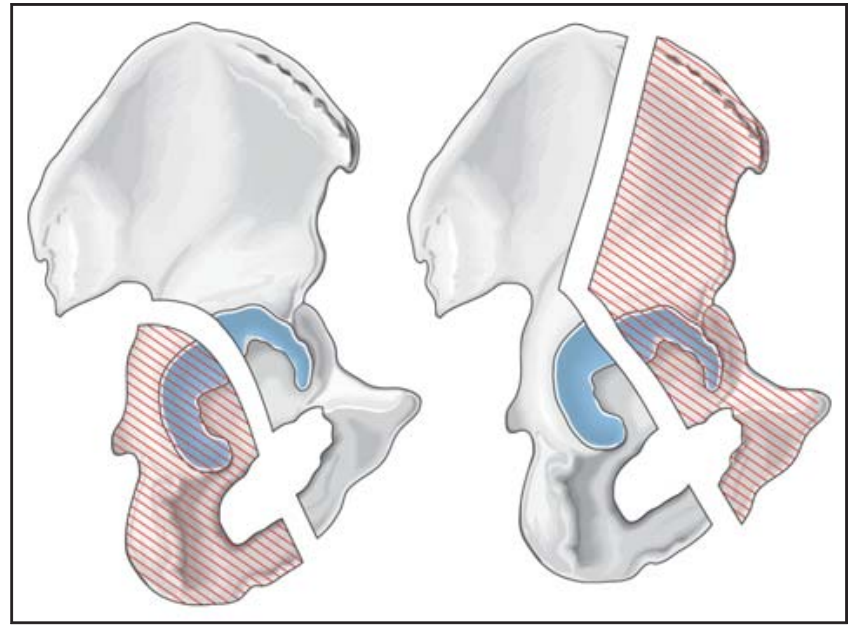

Abb. 7 Hinterer und vorderer Pfeiler des Azetabulums nach Letournel.

Die zusätzliche Computertomografie axial und mit multiplanaren Reformationen liefert die Basis der Detaildiagnostik, insbesondere hinsichtlich prognostisch relevanter Faktoren wie Impressionszonen, intraartikulären Fragmenten und Trümmerzonen sowie zur Feinbeurteilung der Klassifikationstypen.

Die zusätzliche 3-dimensionale Frakturdarstellung vereinfacht häufig die Klassifikation, kann aber zu einem Aufweichen der Detaildarstellung führen.

Anhand der Frakturklassifikation wird der operative Zugang ausgewählt.

\section{Wahl des operativen Zugangs}

Als Standardzugangswege stehen der Kocher-Langenbeck-Zugang sowie der ilioinguinale Zugang zur Verfügung. In seltenen Fällen kommen erweiterte Zugänge und hier v.a. der erweiterte iliofemorale Zugang nach Letournel oder entsprechende Modifikationen zur Anwendung.

Kenntnis der Visualisierbarkeit des knöchernen Beckens über die Standardzugänge.

Der Kocher-Langenbeck-Zugang kann als „Arbeitspferd“ in der operativen Versorgung von Azetabulumfrakturen angesehen werden. Frakturen mit Hauptpathologie im Bereich des hinteren Pfeilers sowie Frakturen mit Querkomponente sind die wesentlichen Frakturtypen, bei denen der Kocher-Langenbeck-Zugang zur Anwendung kommt.

Er erlaubt die visuelle Darstellung des gesamten hinteren Pfeilers und der hinteren Wand sowie von Anteilen des
Pfannendachs. Palpatorisch kann die Innenfläche des Beckens durch das Foramen ischiadicum majus getastet werden (Abb. 9).

Durch zusätzliche Durchführung der chirurgischen Hüftluxation nach Ganz können die Exposition im Pfannendachbereich sowie die intraartikuläre Darstellung deutlich verbessert werden.

Der ilioinguinale Zugang ist der am häufigsten verwendete anteriore Zugang zur Stabilisierung von Azetabulumfrakturen. Die Schwierigkeit bei Anwendung dieses Zugangs ist die nur indirekte Reposition „ohne Sicht" und die entsprechend indirekte Schraubenpositionierung.

Im Wesentlichen kommt er bei Frakturen mit Hauptpathologie im Bereich des vorderen Pfeilers sowie bei Frakturen mit Querkomponente zur Anwendung.

Er erlaubt die visuelle Darstellung der Fossa iliaca, den Bereich der Linea terminalis bis zur Symphyse sowie weiter Anteile der quadrilateralen Fläche. Palpatorisch kann der hintere Pfeiler indirekt im Bereich des Foramen ischiadicum majus sowie an der quadrilateralen Fläche getastet werden (Abb.9). Durch Modifikationen des klassischen Zugangs können weitere Anteile des Beckens visuell dargestellt werden.

Die Problematik des Kocher-Langenbeck-Zugangs sowie des ilioinguinalen Zugangs ist die nur indirekte Darstellbarkeit des kontralateralen Pfeilers. Die Expositionsmöglichkeit ist durch die erweiterten Zugänge deutlich verbessert. Der Basiszugang ist der erweiterte iliofemorale Zugang nach Letournel sowie dessen Modifikationen. 


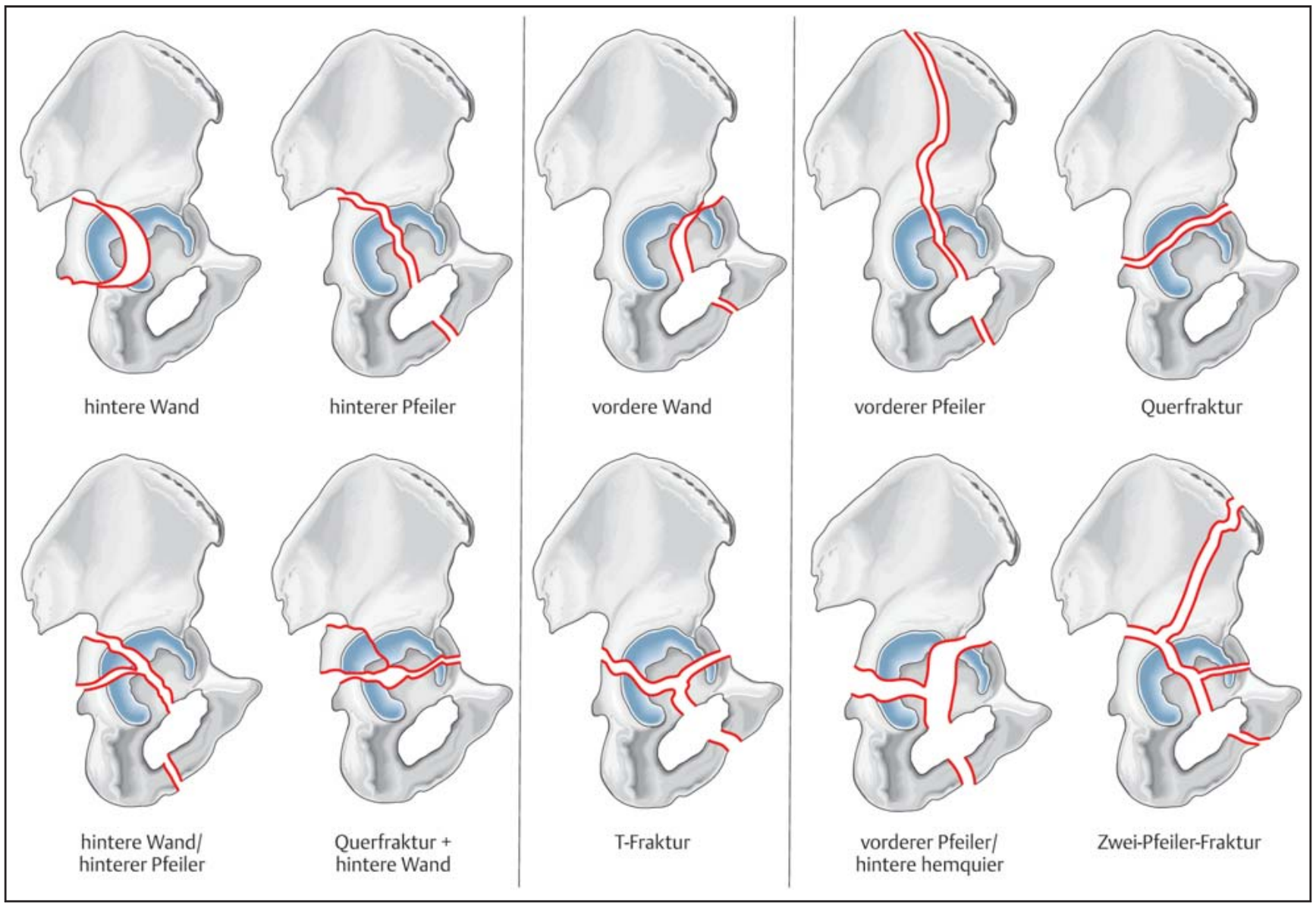

Abb. 8 Klassifikation der Azetabulumfrakturen nach Letournel.

Erweiterte Zugänge kommen bei allen Frakturtypen zur Anwendung, bei denen eine Reposition beider Pfeiler unter Sicht erfolgen muss.

Durch Ablösung der gesamten Abduktoren kann nahezu die gesamte Iliumaußenseite dargestellt werden. Lediglich der Bereich des tiefen vorderen oberen Schambeinasts kann weder palpiert noch eingesehen werden. Auf der Beckeninnenseite ist die Beckenschaufel wie beim 1. Fenster des ilioinguinalen Zugangs einsehbar und die dorsalen Anteile der quadrilateralen Fläche sind palpabel (Abb. 9).

Zusammenfassend kommen folgende Zugänge in Abhängigkeit vom Frakturtyp zur Anwendung (Tab. 1).

\section{Repositionstechniken}

Die anatomische Rekonstruktion von Frakturen des Azetabulums liefert die besten Langzeitergebnisse, obwohl Hinweise bestehen, dass die physiologische Inkongruenz des gesunden Hüftgelenks nicht wieder hergestellt werden kann.
Repositionsplanung bei dorsalen Frakturen unter direkter Sicht beim KocherLangenbeck-Zugang.

Bei den klassischen dorsalen Frakturen des Azetabulums (Fraktur hintere Wand, Fraktur hinterer Pfeiler, Kombination dieser beiden Frakturtypen) ist aufgrund der ausgezeichneten Exposition des Azetabulums über den Kocher-LangenbeckZugang eine exakte anatomische Rekonstruktion relativ einfach. Hier kommen die klassischen Prinzipien der Gelenkrekonstruktion zur Anwendung. Bei Stabilisierung von Frakturen mit Querkomponente kann durch Erweiterung dieses Zugangs zur chirurgischen Hüftluxation auch eine direkte Darstellung der anterioren Gelenkanteile erfolgen.

Repositionsplanung bei anterioren Frakturen nur indirekt beim ilioinguinalen Zugang.

Bei Verwendung des ilioinguinalen $\mathrm{Zu}-$ gangs ist aufgrund der nur indirekten Repositionsmöglichkeit der artikulären Anteile eine spezielle Rekonstruktionstechnik anzuwenden. Hier hat sich die sog. „proximal-to-distal“--Regel bewährt. Dabei erfolgt die Rekonstruktion beginnend im Bereich des Beckenkamms und setzt sich sukzessive bis zur azetabulumnahen Linea terminalis fort. Gerade hier ist auf eine exakte Reposition zu achten, da jeder "Repositionsfehler" sich entsprechend fortleitet und damit im artikulären Bereich zu einer entsprechenden Spalt- oder Stufenbildung führen kann.

Entsprechend ist die Kenntnis der möglichen Repositionsinstrumente entscheidend.

Im Folgenden werden 2 typische Beispiele zur Planung einer ventralen und dorsalen Stabilisierung dargestellt.

Beispiel 1: dorsale Osteosynthese (Abb. 10)

48-jähriger Patient nach PKW-Unfall mit Fraktur des hinteren Pfeilers und zusätzlicher Fraktur der hinteren Wand mehrfragmentär mit zusätzlichen intraartikulären Fragmenten. Die Fraktur ließ sich primär reponieren, jedoch nicht retenie- 


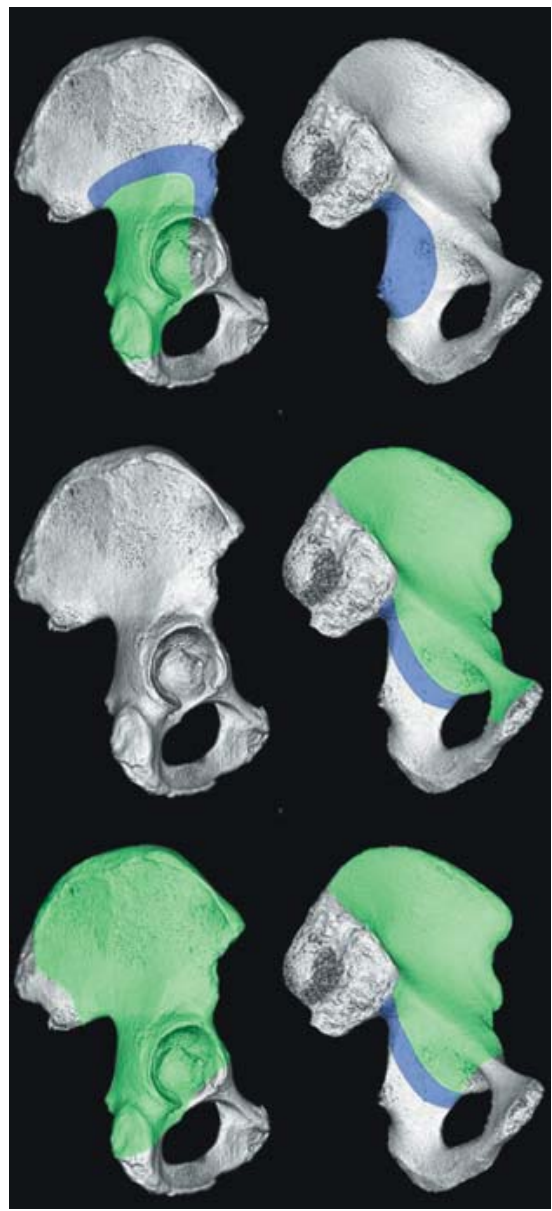

Abb.9 Exposition der typischen Azetabulumzugänge (grün: einsehbar, blau: palpabel): Kocher-Langenbeck-Zugang (oben), ilioinguinaler Zugang (Mitte), erweiterter iliofemoraler Zugang (unten).

ren, sodass die unmittelbare Versorgung erfolgte.

Gemäß präoperativer Planung wurde ein Kocher-Langenbeck-Zugang gewählt. Primär erfolgte die Reposition des hinteren Pfeilers und dessen Fixation mit einem 4-Loch-Plättchen. Danach erfolgte die Reposition der Fragmente der hinteren Wand und Fixation mit Drähten. Abschließend wurde eine dorsale Neutralisationsplatte platziert und mit je 2 Schrauben proximal und distal fixiert.

\section{Beispiel 2: ventrale Osteosynthese (Abb. 11)}

64-jähriger Patient nach Leitersturz. Die radiologische Diagnostik ergab eine hohe Fraktur des vorderen Pfeilers mit Dislokation im Bereich der vorderen Wand und gering dislozierter zentraler Pfannendachimpression.

Tab. 1 Empfohlene Zugänge für die einzelnen Frakturtypen bei Azetabulumfrakturen.

\begin{tabular}{lll} 
& 1. Wahl & Alternativen \\
\hline hintere Wand & Kocher-Langenbeck & - \\
\hline hinterer Pfeiler & Kocher-Langenbeck & - \\
\hline vordere Wand & ilioinguinal & - \\
\hline vorderer Pfeiler & ilioinguinal & iliofemoral \\
\hline Querfraktur & Kocher-Langenbeck & $\begin{array}{l}\text { ilioinguinal } \\
\text { Kocher-Langenbeck + Erweiterung }\end{array}$ \\
\hline hintere Wand + Pfeiler & Kocher-Langenbeck & - \\
\hline quer + hintere Wand & Kocher-Langenbeck & $\begin{array}{l}\text { Kocher-Langenbeck + Erweiterung } \\
\text { erweitert iliofemoral }\end{array}$ \\
\hline T-Fraktur & Kocher-Langenbeck & $\begin{array}{l}\text { Kocher-Langenbeck + Erweiterung } \\
\text { erw. iliofemoral }\end{array}$ \\
& ilioinguinal \\
(erw. iliofemoral) & ilioinguinal & $\begin{array}{l}\text { Kocher-Langenbeck + ilioinguinal } \\
\text { Kocher-Langenbeck + Erweiterung } \\
\text { Stoppa + ilioinguinal (1. Fenster) }\end{array}$ \\
\hline $\begin{array}{l}\text { vorderer Pfeiler + } \\
\text { hintere Hemiquer-Fx }\end{array}$ & ilioinguinal & $\begin{array}{l}\text { erw. iliofemoral } \\
\text { Kocher-Langenbeck + ilioinguinal } \\
\text { Stoppa + ilioinguinal (1. Fenster) }\end{array}$
\end{tabular}

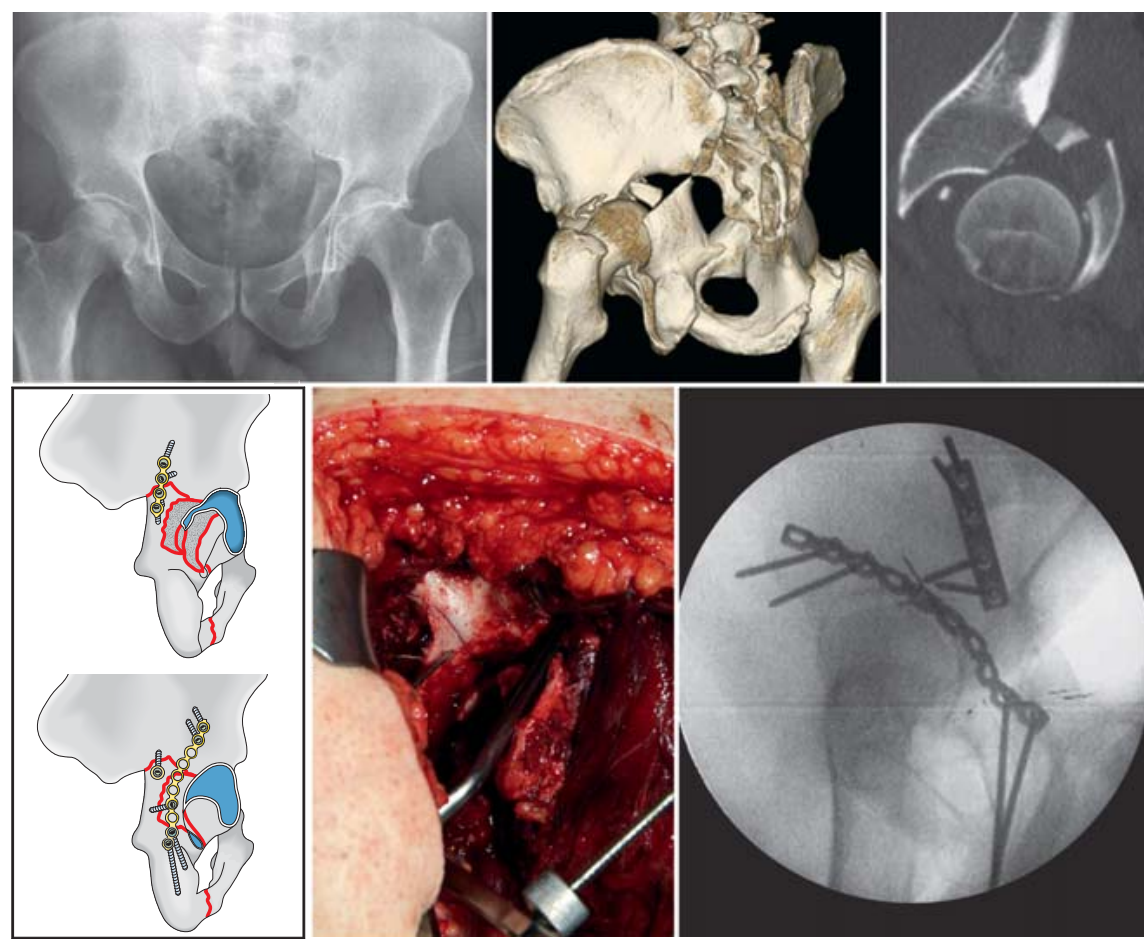

Abb.10 Planung der Osteosynthese einer hinteren Pfeilerfraktur mit Fraktur der hinteren Wand (Details s. Text).

Es wurde der folgende operative Plan erstellt: ilioinguinaler Zugang, Rekonstruktion „proximal-to-distal“, Schraubenosteosynthese Crista iliaca nach Reposition mittels Weller-Zange, ggf. 1/3-Rohr-Plättchen Fossa iliaca, abschließende ilioinguinale Rekonstruktionsplatte auf die Linea terminalis.
Das postoperative Röntgenbild zeigt die exakte Umsetzung der präoperativen Planung mit anatomischer Gelenkrekonstruktion. 


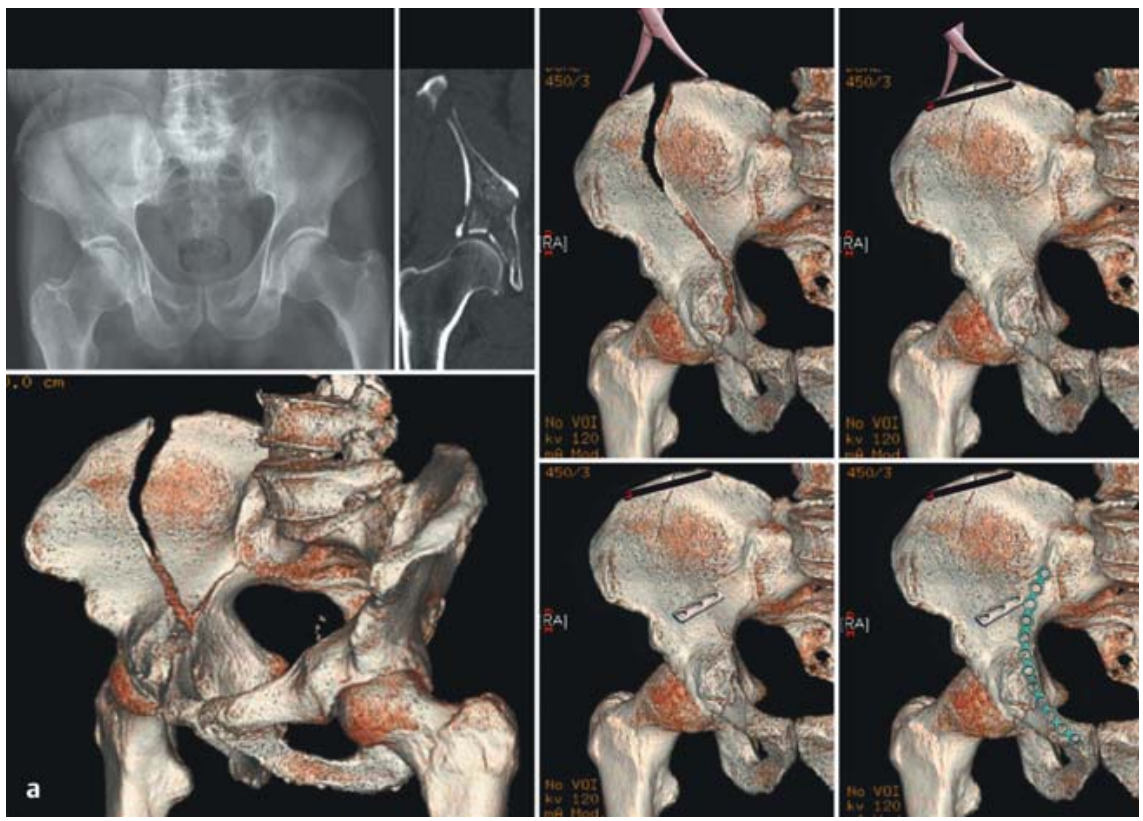

Abb. 11a Planung der Osteosynthese einer vorderen Pfeilerfraktur (Details s. Text).

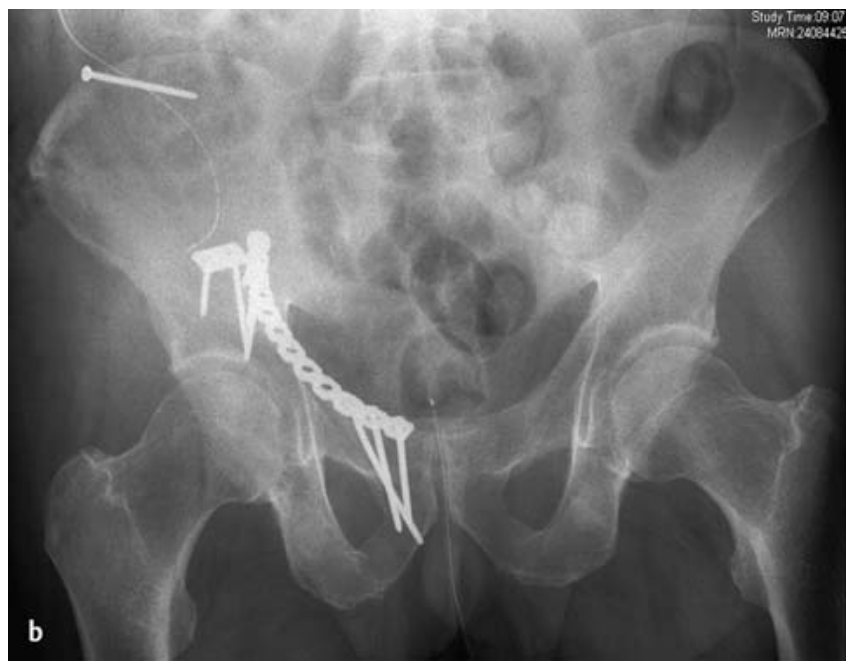

Abb. 11 b Postoperatives Ergebnis mit umgesetzter Planung.

\section{Literatur}

${ }^{1}$ Cimerman M, Kristan A. Preoperative planning in pelvic and acetabular surgery: the value of advanced computerised planning modules. Injury 2007; 38: 442-449

2 Gänsslen A, Pohlemann T, Krettek C. [A simple supraacetabular external fixation for pelvic ring fractures]. Oper Orthop Traumatol 2005; 17: 296-312
${ }^{3}$ Gänsslen A, Pohlemann T, Krettek C. [Internal fixation of sacroiliac joint disruption]. Oper Orthop Traumatol 2005; 17: 281-295

${ }^{4}$ Gänsslen A, Hufner T, Krettek C. Percutaneous iliosacral screw fixation of unstable pelvic injuries by conventional fluoroscopy. Oper Orthop Traumatol 2006; 18: 225-244

5 Gänsslen A, Krettek C. [Internal fixation of acetabular both-column fractures via the ilioinguinal approach]. Oper Orthop Traumatol 2009; 21: 270-282
${ }^{6}$ Gänsslen A, Steinke B, Krettek C. [Internal fixation of acetabular posterior wall fractures]. Oper Orthop Traumatol 2009; 21: 283-295

7 Hefzy MS et al. Kinematics of the human pelvis following open book injury. Med Eng Phys 2003; 25: 259-274

8 Helfet D, Bartlett C, Lorich D. The use of a single limited posterior approach and reduction techniques for specific patterns of acetabular fractures. Op Tech Orthop 1997; 7: 196-205

${ }^{9}$ Letournel E. Annotation to pelvic fractures. Injury 1978; 10: 145-148

${ }^{10}$ Letournel E, Judet R. Fractures of the Acetabulum. 2nd ed. Berlin, Heidelberg, New York: Springer; 1993

${ }^{11}$ Leung K et al. Operative treatment of unstable pelvic fractures. Injury 1992; 23: 31-37

12 Müller M. CCF Comprehensive Classification of Fractures. Bern: M.E. Müller Foundation; 1996

13 Orthopaedic Trauma Association. Fracture and dislocation compendium. J Orthop Trauma 1996; 10: 71-75

14 Pohlemann T, Tscherne H. Fixation of sacral fractures. Op Tech Orthop 1995; 9: 315-326

15 Pohlemann T. Pelvic Ring Injuries: Assessment and Concepts of surgical Management. In: Rüedi TP, Murphy WM, eds. AO Principles of Fracture Management. Stuttgart, New York: Thieme; 2000: 391-414

${ }^{16}$ Pohlemann T, Gänsslen A, Tscherne H. Sacral Fractures. In: Tile M, Helfet DL, Kellam JF, eds. Fractures of the Pelvis and Acetabulum. 3rd ed. Philadelphia: Lippincott Williams \& Wilkins; 2003: 294-322

${ }_{17}$ Tile $M$. Pelvic ring fractures: should they be fixed? J Bone Joint Surg [Br] 1988; 70: 1-12

18 Tile M, Helfet DL, Kellam JF. Fractures of the Pelvis and Acetabulum. Lippincott, Williams and Wilkins; 2003

19 Tscherne H, Pohlemann T. Tscherne Unfallchirurgie: Becken und Acetabulum. Berlin, Heidelberg, New York: Springer; 1998

\section{Dr. med. Axel Gänsslen Oberarzt}

Klinik für Unfallchirurgie, Orthopädie und Neurotraumatologie

Allgemeines Krankenhaus Celle Siemensplatz 4 29223 Celle

dr.gaensslen@gmx.de

Prof. Dr. med. Hans-Jörg Oestern Schubertstraße 12 29223 Celle 